\title{
Rhythmic recurrency in dance to music with ambiguous meter
}

\section{Sofia Dahl}

Aalborg University Copenhagen

Copenhagen, Denmark

sof@create.aau.dk

\author{
George Sioros \\ RITMO, University of Oslo \\ Oslo, Norway \\ georgios.sioros@imv.uio.no
}

\section{ABSTRACT}

Head movements of groups of participants moving to music allowing for both duple and triple subdivisions were analysed using Recurrence Quantification Analysis. As expected the maximum recurrence rate varied between participant, position in the music, and between groups. The lags of maximum recurrence were only partly corresponding to the metrical levels of the music and syncopated sections confused participants so that they moved to $4 / 4$ rather than $6 / 8$.

\section{CCS CONCEPTS}

- Applied computing $\rightarrow$ Sound and music computing; Psychology; Sociology; •Information systems $\rightarrow$ Physical data models;

\section{KEYWORDS}

dance, embodiment, recurrence analysis,entrainment, meter, metric subdivision

*also visiting associate professor at Department of Musicology, University of Oslo.

Permission to make digital or hard copies of part or all of this work for personal or classroom use is granted without fe provided that copies are not made or distributed for profit or commercial advantage and that copies bear this notice and the full citation on the first page. Copyrights for third-party components of this work must be honored. For all other uses, contact the owner/author(s)

MOCO'18, June 2018, Genoa, Italy

C 2016 Copyright held by the owner/author(s).

ACM ISBN 123-4567-24-567/08/06 . \$15.00

https://doi.org/10.475/123_4 


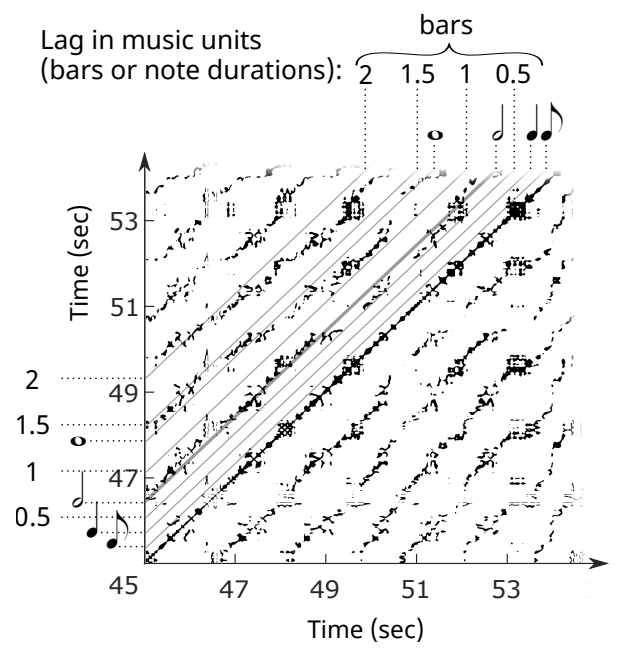

Figure 1: An example of a Recurrence Plot calculated for the first participant in Group 2. The first 10 s of the 20 s time window is shown (at 45-55 $s$ in the musical excerpt). Each black dot correspond to a state recurring with a specific time lag indicated by its distance from the main diagonal. Some example of time lags corresponding to different metrical and rhythmical levels in the music are indicated on the axes. The maximum Recurrence Rate (RR) for the 20s window is observed at a lag of $1.46 \mathrm{~s}$, which is found within $5 \%$ of the half note duration at $1.42 \mathrm{~s}$. Since we are interested in periodicities of the metrical hierarchy and in order to exclude the main diagonal which is always present in auto-RP, only time lags between $0.23 \mathrm{~s}$ and $4.50 \mathrm{~s}$ were considered in the calculations of the maximum RR.

\section{INTRODUCTION}

Recently, spontaneous movements to music have gained research interest and studies have investigated the overall movement patterns in relation to music features (e.g. [1]). If the beat level, tactus, is clear and the metrical accents unambiguous we can expect that movements would emphasize the hierarchical structures (see e.g. [11]). We here investigate a case where the musical rhythm is more ambiguous, leaving room for different interpretations of metrical accents, looking at how dancers settle for a metrical level to entrain [4] with in a group setting.

One approach to analyze coupled behaviour between participants from time-series data is Recurrence Quantification Analysis (RQA) [3, 8, 10]. In RQA, the self-similarity of patterns in time-series can be investigated despite the (sometimes complex) patterns recurring irregularly or in different time-scales [6]. Thus, RQA offers an attractive alternative to traditional time-series analysis methods such as auto- or cross correlation.

A recurrence plot $(\mathrm{RP})$ resembles a similarity matrix, where the state of a system at each moment in time is compared to the states at all other moments in time. States are commonly defined as vectors consisting of a number points of the same time series which have some delay between them. In this way, a one-dimensional signal can be embedded into a multidimensional phase-space. The states $(i, j)$ are considered similar if their distance is below a certain threshold.

The main diagonal of a RP corresponds to pairs of states that occur simultaneously, while any other line parallel to the main diagonal correspond to states occurring with a time lag. That is, a parallel to the diagonal describes the recurrence of a system after a certain time (resembling the lags for autoor cross correlation functions). In this way we can observe and measure periodicities within a system. An example of a RP can be seen in Figure 1. The recurrence rate (RR) is a way of quantifying how much the states of a system recur and is defined as the ratio of the number of the pairs of similar states to the total number of pairs (i, j). The RR can be calculated for the entire plot, a part of it, or for a diagonal.

In this work, we explore how RQA can be applied to detect recurrent movement patterns in the case of participants moving to music with ambiguous metric subdivision. We hypothesize that participants will share similar hierarchical subdivisions toward the end of the music stimulus, reflected in higher recurrence rates for these lags.

\section{EXPERIMENT}

A 3 minute long excerpt from Samurai Hee-Haw (Mark Johnson, "Bass Desires") was chosen due to the rhythm being inherently ambiguous with respect to its metrical (hierarchical) interpretation The time signature of the piece is in $6 / 8$ and can be perceptually subdivided as duple or triple. The section chosen (starting from $30 \mathrm{~s}$ ) begins with a bass vamp repeating; a guitar melody follows and 


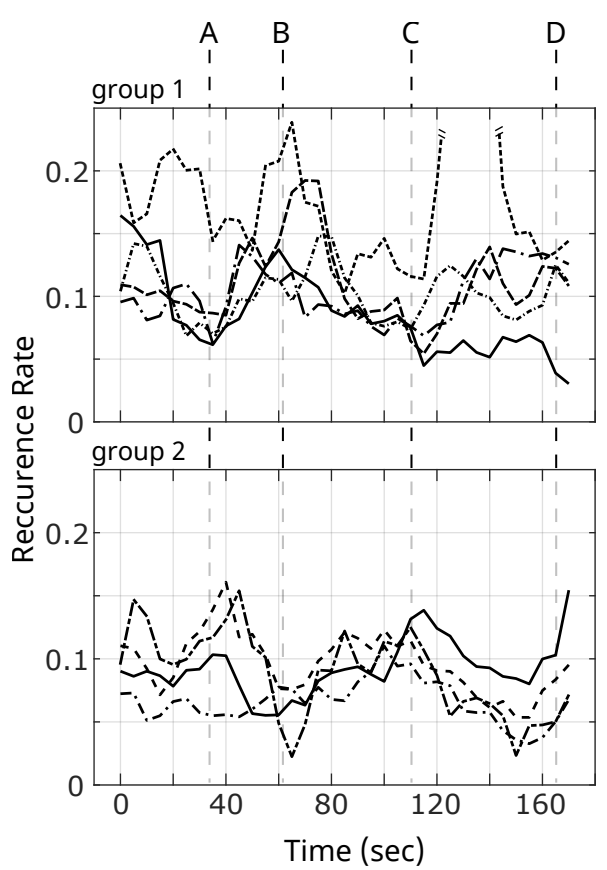

Figure 2: Maximum Recurrence Rate across a diagonal for each participant for Group 1 (top panel) and Group 2 (bottom panel). Each point in the curves shows the maximal recurrence rate along a diagonal in a RP. RPs were calculated for windows of 2000 samples (duration of 20 s) with a hop size of 500 samples $(5 \mathrm{~s})$ resulting in $35 \mathrm{RPs}$ for each participant. The dashed vertical lines mark specific points in the musical excerpt: A melody enters; $B$ repetition of melody; $C$ highly syncopated part in solo; $\mathrm{D}$ melody repeats final time. is repeated once before progressing into a guitar solo where the rhythmical markings of the drums become increasingly syncopated; finally the guitar melody is repeated once more before the excerpt ends at $3 \mathrm{~min} 38 \mathrm{~s}$.

In all 13 (1 female) participants, (MSc. students at Aalborg University partaking in a course on Embodied interaction) were tested in groups of four or five at a time. We here restrict our analysis to data from Groups 1 and 2, with five and four participants respectively.

Participants were invited into the capture volume of an Optitrack motion capture system, equipped with caps with reflective markers attached, and instructed to stand in a circle so that they were facing each other and "Synchronize with the music". The choice of measuring head movements only was motivated by these generally reflecting overall locomotion and weight shift, (c.f.[7]), and because the vestibular system has been shown to be of importance for how we perceive rhythm [9]. Thus, movements of the head can be assumed to both reflect overall body movement to an observer and also represent the input to the vestibular system of the dancers themselves. The recording and playing of the musical stimulus was controlled via a game engine (Unity). Data was recorded at $100 \mathrm{~Hz}$, stored as a text file and imported to Matlab.

\section{ANALYSIS}

The marker position data was inspected and some brief outliers were cleaned out and interpolated using the MoCap toolbox [2]. The same toolbox was used to filter the position data ( $2^{\text {nd }}$ order Butterworth LP-filter, $0.2 \mathrm{~Hz}$ cutoff), and to calculate velocity and acceleration (both filtered with Savitzky-Golay FIR smoothing filter, 7 and 9 point windows for velocity and acceleration respectively) The subsequent analysis was based on the magnitude of the velocity vector (calculated as the square root of the summed and squared velocities for each dimension) for each participant's head marker, and the acceleration vector for the vertical direction only.

The main part of the analysis used crptoolbox for Matlab [8], while the parameters for the RPs (including the similarity thresholds) were derived using the function optimizeParam from the crqa R-package [5], resulting in 2 or 4 embedded dimensions with a delay between 2 and 19 samples for the velocity magnitude data. In other words, the phase space was constructed from two or four copies of the velocity time series each delayed by 2 to 19 samples. We used the euclidean distance as an absolute (self-)similarity metrics for continuous variables.

Since vertical acceleration can be assumed to have a close relationship with participants' locomotion [7] as well as vestibular sense [9], we hypothesized that the recurrence would also be driven by acceleration in the vertical plane. Therefore, in addition to the simple RPs for the velocity magnitude, we used a more hypothesis driven definition of recurring states by combining the velocity magnitude data with vertical acceleration. To this end, we constructed RPs for velocity and acceleration separately, with no embedding dimensions, while the similarity thresholds were derived from the crqa R-package [5] 


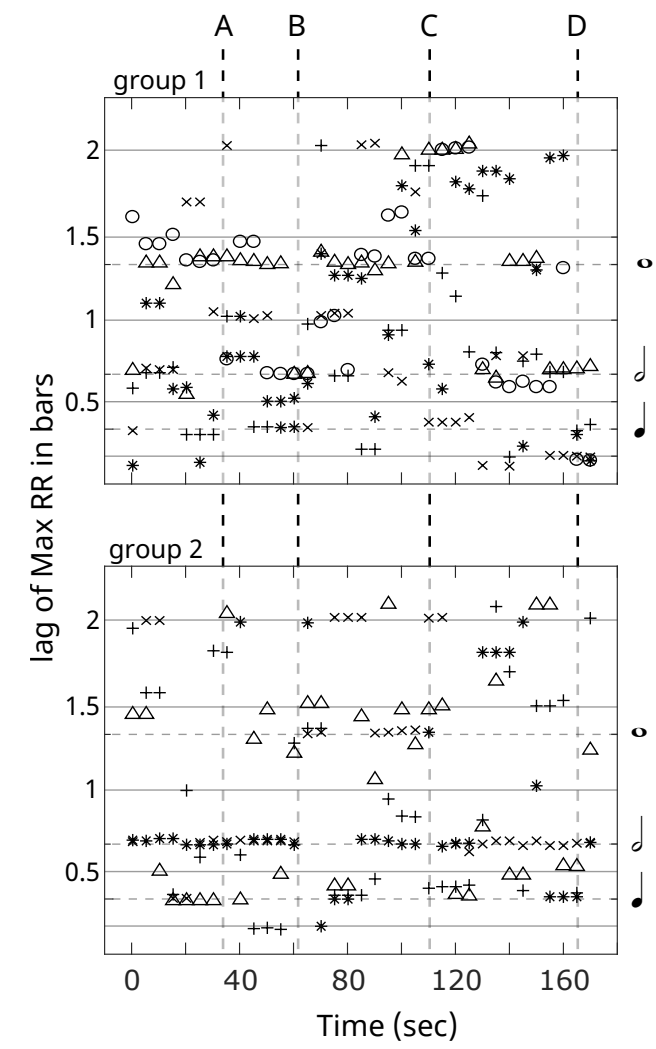

Figure 3: Lags for Maximum Joint Recurrence Rate calculated across each window for individual participants (different shapes) in each group (top panel: group 1; bottom panel: group 2). The horizontal lines mark musically relevant periodicities in the music: Solid lines (main grid) correspond to the periodicities for a $6 / 8$ meter (8th note, half bar, bar and 2 bars); dashed lines correspond to a misinterpretation of the meter as a 4/4 (quarter-, halfand whole note durations). As in Figure 2, different sections of the music are indicated by vertical dashed lines. independently for each. We then created joint-RPs by element-wise multiplication of the independent RPs. In this way, the recurring states were determined by both velocity magnitude and vertical acceleration.

For the quantification analysis of the RPs and joint-RPs, we calculated the RRs across diagonal lines with time lags from the main diagonal between $230 \mathrm{~ms}$ to $4500 \mathrm{~ms}$ (see Figure 1 for one example). We extracted the maximum RRs and the corresponding lags for each participant. The results are shown in Figures 2 and 3 and are discussed in detail in the following section.

\section{RESULTS}

Although the instructions quite intentionally did not mention dancing, all participants interpreted the task in this way. Some participants expressed that at times synchronizing with the music was difficult.

Figure 2 shows the max recurrence rate of the velocity magnitude RPs per window for each of the participants in Group 1 (top) and 2 (bottom). As can be seen in the figure, the maximum recurrence rate is varying throughout the piece. Participant 1 in group1 (top dashed line Figure 2) displays the highest values of recurrence, but a general pattern of peaks and valleys can be discerned in all participants. The pattern is common between participants of the same group but different between the groups. The relationship between the RP windows and the structure of the music excerpt is indicated by vertical dashed lines with letters A-D. At the start, most participants show a recurrence rate of about $0.1-0.15$ and when the melody first is introduced (A, $36 \mathrm{~s}$ ) three of four participants in Group 2 have a peak in recurrence rate, while four participants in Group 1 show a minimum. As the melody is repeated (B $62 \mathrm{~s})$ the pattern appears reversed between the groups. In section C, around $110 \mathrm{~s}$, the drums are very syncopated and Group 1 display a decrease in recurrence around this section, while Group 2 appear to be stable around or above 0.1 . As the melody returns for a third time (D, $166 \mathrm{~s})$ all participants except one increase their recurrence rate.

The maximal RR in the joint velocity-acceleration RPs showed an overall behaviour along the music excerpt very similar to that of Figure 2 but with less noise and lower values $(<0.1$ for most participants). The lower values were expected, as joint RPs impose a stricter requirement for the recurrence state in both velocity magnitude and vertical acceleration. However, the overall group behaviour from Figure 2 remains: The recurrence in relation to the different music sections (A-D) show the same peaks and valleys confirming our assumption that the vertical direction of movement appears to be an important factor in the participants movements. Since the results of the two methods are similar, we continue our analysis with the more data driven hypothesis of joint RPs.

Figure 3 shows the lags for the joint maximum recurrence rate and their relationship to the metrical level of the music at the bar level (horizontal lines). As can be seen, the lags of joint maximum recurrence appear to vary considerably both within and between participants. As a simple measure 
Table 1: Percentage of the lags for maximum joint recurrence of Figure 3 falling within a $5 \%$ deviation from a metrical level.

\begin{tabular}{lcc}
\hline Metrical Levels & Group 1 & Group 2 \\
\hline All metric levels & 60 & 70 \\
Belonging to 6/8 or 3/4 & 29 & 34 \\
Half and Whole note duration & 31 & 36 \\
\hline
\end{tabular}

Table 2: Comparison between section $C$ (RP windows from 120 to $145 \mathrm{~s}$ ) and the remainder of the music excerpt (sections A, B and D together) of the percentage of the time-lags that fall on metrical levels.

\begin{tabular}{lcc}
\hline Section & Group 1 & Group 2 \\
\hline A, B \& D & 64 & 74 \\
C & 30 & 42 \\
\hline
\end{tabular}

of level of entrainment we calculated the percentage of all maximum recurrence point-lags that fall within $5 \%$ deviation from one of the metrical levels indicated in Figure 3. Table 1 shows that by this criteria, $60-70 \%$ of the maximum recurrence point-lags of the participants' movements fall on one of the possible metrical levels. However, only $29-34 \%$ of the lags indicate that participants moved in one of 6/8 or 3/4 meter. By comparison, 31-36\% of lags were half and whole note durations. Thus, although the participants movements were indicating a metrical level for the greater part of the music stimulus, they appear to have misinterpreted the meter for more than half of the time.

Comparing the distribution of time-lags for different parts of the music excerpt the results vary considerably. A summary of the comparison is seen in Table 2. Section C seems to have been rather disorienting for most of the participants as the percentage of lags corresponding to metrical levels is roughly halved for that section compared to sections $\mathrm{A}, \mathrm{B}$ and $\mathrm{D}$.

\section{DISCUSSION}

The results are promising in terms of RQA as a useful method to detect recurrent movement patterns when moving to music with ambiguous metric subdivision. Even though the participants were not doing the same type of movements, they were all repeating themselves in a similar way within their group. Their repetition were partly following structure of the music, but also emphasizing periods not part of the metrical hierarchy.

It appears that the complexity of the musical excerpt used for the experiment, especially the emphasized syncopated bass vamp and the increased rhythmical complexity during the solo part, confused the participants. When comparing the percentage of maximum joint recurrence lags corresponding to metrical levels during the syncopated part of the solo $(C)$ with those during the last repetition of the melody (D) it appears that the drum synchopation in the solo part was particularly confusing for participants. The third repetition of the melody increased the max recurrence rate, which is some support for our hypothesis of shared metrical structure at the end.

In this short paper we aimed at exploring a group behavior and not of participants' direct imitation of each others movements. Our analysis focused on the way the participants repeated themselves as a group, regardless of whether their movement was unique or not. The group behavior was reflected in the observation of periods of higher repetition common between the participants of the same group but not between participants of different groups. A more detailed exploration of the interaction between participants requires the analysis of cross-RPs for pairs of participants similar to [5]. The maximum RR across diagonals and the corresponding lags can reveal specific interactions, for instance one participant imitating another as indicated by a high cross-RR between two participants combined with a lag from the main diagonal. 


\section{CONCLUSION}

Our results so far indicate Recurrence Quantification Analysis as a promising tool to detect underlying patterns of complex non-linear couplings within and between participants interacting with musical stimuli - a task characterized of complexity on many levels. In general, the participants within a group displayed the same overall pattern of points of higher and lower maximum recurrence coinciding with musical sections. However, these patterns differed between groups. Interestingly, the lags of maximum joint recurrence only partially corresponded to the metrical levels in the music and in many cases the meter was interpreted by participants as $4 / 4$ rather than $6 / 8$. A music stimulus with a simpler rhythmical structure is likely to have produced more clear results.

\section{AUTHOR CONTRIBUTION}

Author Dahl conceived, prepared and performed the experiment and pre-processed data, while the major part of the RQA analysis was performed by author Sioros. Both authors contributed equally in writing the manuscript.

\section{REFERENCES}

[1] Birgitta Burger, Marc R Thompson, Geoff Luck, Suvi Saarikallio, and Petri Toiviainen. 2013. Influences of rhythm-and timbre-related musical features on characteristics of music-induced movement. Frontiers in psychology 4 (2013).

[2] Birgitta Burger and Petri Toiviainen. 2013. MoCap Toolbox-A Matlab toolbox for computational analysis of movement data. In 10th Sound and Music Computing Conference, SMC 2013, Stockholm, Sweden. Logos Verlag Berlin.

[3] Federica Censi, Giovanni Calcagnini, and Sergio Cerutti. 2015. Dynamic coupling between respiratory and cardiovascular system. In Recurrence Quantification Analysis: Theory and best practices, Jr. C. L. Webber and N. Marwan (Eds.). Springer $195-212$.

[4] Martin Clayton. 2012. What is Entrainment? Definition and applications in musical research. Empirical Musicology Review 7, 1-2 (2012), 49-56.

[5] Moreno I Coco and Rick Dale. 2014. Cross-recurrence quantification analysis of categorical and continuous time series: an R package. Frontiers in psychology 5 (2014).

[6] Alexander P Demos, Roger Chaffin, and Topher Logan. 2017. Musicians body sway embodies musical structure and expression: A recurrence-based approach. Musicae Scientiae (2017), 1029864916685928.

[7] Hamish G MacDougall and Steven T Moore. 2005. Marching to the beat of the same drummer: the spontaneous tempo of human locomotion. Journal of applied physiology 99, 3 (2005), 1164-1173.

[8] Norbert Marwan, M Carmen Romano, Marco Thiel, and Jürgen Kurths. 2007. Recurrence plots for the analysis of complex systems. Physics reports 438, 5 (2007), 237-329.

[9] Jessica Phillips-Silver and Laurel J Trainor. 2007. Hearing what the body feels: Auditory encoding of rhythmic movement. Cognition 105, 3 (2007), 533-546.

[10] Kevin Shockley and Michael A Riley. 2015. Interpersonal couplings in human interactions. In Recurrence Quantification Analysis: Theory and best practices, Jr. C. L. Webber and N. Marwan (Eds.). Springer, 399-421.

[11] Petri Toiviainen, Geoff Luck, and Marc R Thompson. 2010. Embodied meter: hierarchical eigenmodes in music-induced movement. Music Perception: An Interdisciplinary Journal 28, 1 (2010), 59-70. 